\title{
Correspondence
}

\section{Bone marrow biopsy specimens in assessment of remission in acute leukaemias}

We found the paper on the value of bone marrow biopsy in acute leukaemias interesting. ${ }^{1}$ We have found that biopsy specimens are more informative regarding cellularity, blast cell percentages and their response to chemotherapy. We have also attempted to determine the usefulness of trephine biopsy specimens for assessing remission in acute leukaemias: one third of the marrow aspirates have been inconclusive in our hands. We carried out marrow trephine biopsies and aspirates in 15 patients with acute myeloid leukaemia (AML) and 20 patients with acute lymphoblastic leukaemia seven days after completion of chemotherapy ${ }^{2}$ in cases of AML, and at the end of four or six cycles of induction chemotherapy in cases of ALL. ${ }^{3}$ Twelve (34.3\%) of the aspirates were "unsatisfactory"; all the biopsy specimens were adequate for comment. Based on the histomorphology of the biopsy specimens, 15 cases were in remission, 19 cases had blast cell transformation and one case displayed myelonecrosis.

Fifteen cases in remission showed pronounced hypocellularity, marrow oedema, serous atrophy with evidence of fat, erythroid, and megakaryocytic regeneration ${ }^{4}$ in four cases. Nine of $12(75 \%)$ of the "unsatisfactory" aspirates came from these cases because induction chemotherapy depletes the marrow of leukaemic cells, resulting in hypocellularity and oedema ${ }^{5}$; the core biopsy specimens showed evidence of remission.

Nineteen cases showed the presence of blast cells in the marrow trephine specimen with normal to hypercellularity in 15 and hypocellularity in four cases. Sixteen of 19 $(84 \cdot 2 \%)$ of the aspirates in this group were "unsatisfactory" because of good marrow cellularity. This varied in the biopsy specimens in different marrow spaces and blast cell distribution was not uniform. Because blast cells are present only in some marrow spaces, the aspirate may not include such a focus resulting in overdiagnosis of remissions. This was true of three cases which were reported as being "in remission" according to the marrow aspirate, but trephine biopsy specimens showed patchy clustering of blasts. Core marrow biopsy seems, therefore, to be essential for precise assessment of remission after induction chemotherapy.

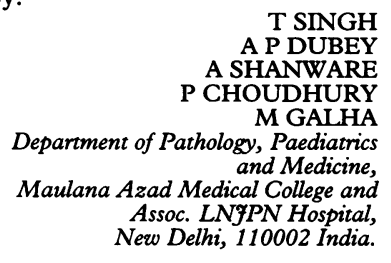

1 Winfield DA, Polacarz SV. Bone marrow histology 3: Value of bone marrow core biopsy in acute leukemia myelodysplastic syndromes and chronic myeloid leukemia. $\mathfrak{f}$ Clin Pathol 1992;45:855-9.

2 Priesler HD, Rustum Y, Henderson ES, et al. Treatment of acute non-lymphocytic binoside induction therapy and comparison binoside induction therapy and comparison
of two maintenance regimes. Blood 1979;53: 455-64.
3 Miller DR, Leiken S, Albo V, Sather H, Karen M, Hammond D. Prognostic factors and therapy in acute lymphoblastic leukemia of childhood. Cancer 1983;51:1041-9.

4 Islam A, Catovsky D, Galton D. Histological study of bone marrow regeneration following chemotherapy for Acute myeloid
leukemia and chronic granulocytic leukemia leukemia and chronic granulocytic leukemia 45:535-40.

5 Roberts MM, Juttner CA, Kimbler BK. Bone marrow biopsy during induction chemotherapy for Acute myeloid leukemia identifies only $50 \%$ of patients with resistant disease. Leuk Res 1988;12:817-21.

\section{Dr Winfield comments:}

The comments of Dr Singh and his colleagues on the value of bone marrow core biopsy specimens in assessing remission in acute leukaemia reinforce the observations in our paper that core biopsy specimens provide a more accurate assessment of total marrow cellularity and of blast cell numbers. Their letter also raises the question of when marrow samples should be taken after chemotherapy to assess remission and whether both marrow aspirate and trephine core biopsy samples are required.

For the current Medical Research Council AML 10 Trial, the policy is that marrow aspirate should be taken two weeks after the completion of chemotherapy. If the aspirate is normocellular, contains less than $5 \%$ leukaemic blast cells, and shows evidence of normal maturation of other marrow elements then the patient is in complete remission. If the marrow fragments and trials are hypoplastic then an aspiration should be repeated in one week. Only if the marrow is diluted with peripheral blood and contains no fragments is a trephine biopsy indicated.

The current MRC Acute Lymphoblastic Leukaemia Trial (UKALL XII) operates a similar policy, using day 22 after the start of remission induction as the time for assessment. The use of these protocols will usually enable the haematologist to provide a reasonably accurate assessment of remission, and I cannot therefore agree with Dr Singh's conclusion that marrow core biopsy is essential for assessing remission in all cases of acute leukaemia. Selective use of trephine biopsies has the additional advantage for many patients of avoiding the discomfort and haemorrhagic problems which may be associated with this procedure.

\section{Histopathology EQAs: the definition} and identification of poor performance

Dr Furness and Professor Lauder must be complimented on the development of a scoring system for histopathology EQAs. ${ }^{1}$ Schemes based on this sort of assessment should provide valuable educational feedback and are likely to become an essential element in continuing medical education for histopathologists. The authors admit, however, that the programme does not attempt to define what is an unacceptable or dangerous level of performance, but state that this is a problem that the profession as a whole must address. While the question of what to do about poor performance may be decided by a dialogue between the profes- sion, accreditation authorities, and managers, I submit that definition and identification of inadequate performance is a problem that individual schemes must tackle as only they will have the appropriate information available to them.

While trying to organise a local EQA scheme that aims to use Furness's computer programme I have proposed a way of defining and identifying inadequate performance that may be of interest to others. Briefly, it is proposed to form a committee of four to six participants of the EQA scheme who would meet once a year to discuss in detail the accumulated responses of those participants denoted by the marking system as having low scores or having given a number of dangerous diagnoses. The response sheets would be identified by a code number and if necessary retyped to ensure anonymity. This "standards committee" will then decide by consensus whether any set of replies constitutes inadequate performance for someone responsible for surgical histopathology reporting and if so what type of "Dear colleague" letter to send. This proposal has the advantage that the standard is set by the scheme participants and not imposed by an outside authority: as a result it appears locally acceptable provided that committee members are representative (rotation of members has been suggested).

Should other EQA schemes adopt a similar method of assessment then comparability of thresholds for action between schemes could be facilitated by exchange of standards committee members or by the inclusion of a representative from an accrediting authority. It is only by the use of such schemes that the profession can assure clinicians, managers, and the public that our standards are consistently high.

$$
\begin{array}{r}
\text { DFR GRIFFITHS } \\
\text { Department of Pathology, } \\
\text { University of Wales College of Medicine, } \\
\text { Heath Park, } \\
\text { Cardiff CF4 4XN }
\end{array}
$$

1 Furness PN, Lauder I. Response analysis in histopathology external quality assessment schemes. F Clin Pathol 1993;46:357-63.

Dr Furness and Professor Lauder comment:

We are grateful for the encouraging comment and constructive criticism provided by Dr Griffiths. His suggestion of a local "standards committee" is certainly one way forward. Such a committee could also review other performance parameters, such as measurements of the timeliness of reports. Local knowledge will certainly be needed to evaluate results before any drastic remedial action is taken; for example, one should not censure a pathologist for poor performance in urological pathology if he has worked for 20 years at a hospital where urological surgery is never carried out.

We consequently agree that the control of "standards committees" must be kept with local pathologists who understand the local situation. Nevertheless, it is obvious that if such committees are to have any credibility or consistency they will have to work to clear national guidelines, and they will have to have well defined "teeth". The problem of defining persistent poor performance is therefore not completely solved. Our understanding is that the histopathology Advisory Panel of the Joint Working Group on Quality Assurance is considering such guidelines, and we suggest that $\mathrm{Dr}$ Griffiths' helpful letter is copied to the chairman of the panel, Professor F Walker. 